\title{
Context-Aware Interaction Models in Cross-Organizational Processes
}

\author{
Florian Skopik, Daniel Schall, Schahram Dustdar \\ Distributed Systems Group \\ Vienna University of Technology \\ Argentinierstraße 8/184-1, A-1040 Vienna, Austria \\ \{skopik|schall|dustdar\}einfosys.tuwien.ac.at
}

\author{
Michele Sesana \\ TXT e-solutions S.p.A. \\ Via Frigia 27, 20126 Milano, Italy \\ michele.sesana@txt.it
}

\begin{abstract}
Rigidly pre-planned business processes are applied in the field of production planning and product development to coordinate the collaboration of single enterprises. Each step in these workflows is precisely scheduled, accounting for external constraints such as availability of material, delivery dates, and efficiency of humans and machines. However, finally all these steps are performed, or at least controlled, by humans and it is likely that in human-operated environments failures happen, and misunderstandings require adaptations and adhoc interference to avoid delays in workflow executions.

In this paper, we discuss the role of human interaction support in traditional process-oriented environments, and present new approaches to dynamic involvement and interactions with collaboration partners. We highlight a typical use case where human experts are flexibly involved in certain steps of workflows that assist single tasks owners to solve emerging problems. In our approach, experts are discovered based on dynamically changing contextual constraints, such as problem areas and required expertises, and enable their fast involvement by using Web 2.0 communication facilities.
\end{abstract}

Keywords-Context-aware collaboration, dynamic expert involvement, cross-organizational process models, trust

\section{INTRODUCTION}

Small and medium-sized companies create alliances to compete with global players, to cope with the dynamics of economy and business, and to harvest business opportunities that a single partner cannot take. In such networks where companies, communities, and individuals form virtual organizations (VOs) [1], enterprise collaboration support has been a major research track. A virtual enterprise is a temporary alliance of enterprises that come together to share skills or core competencies and resources in order to better respond to business opportunities, and whose cooperation is supported by computer networks [1].

A process in a VO spans multiple organizations, whereas each task is either performed by only one physical company or processed by various partners. While the interfaces and flow of information and goods between the single task owners are pre-planned, human interactions are usually not static. Especially in those cases where processes have not been executed several times; thereby providing historical information, need dynamic interactions of participants to adapt and optimize workflows, or to solve unforeseen problems. In such scenarios we distinguish between two funda- mental kinds of human interactions: (i) organization-internal interactions, such as the communication and coordination between members of the same company; and (ii) crossorganizational interactions that take place between different physical companies.

Typical research challenges that arise in such scenarios [2] deal with the discovery of people in partner organizations, accounting for contextual constraints (online presence state, contact details, preferred communication channels), personal profiles (skills, certificates, collected experiences), and personal relations based on previous joint collaborations in similar situations. Figure 1 depicts a typical scenario, where the task "Mechanical Specification" of a construction process is jointly performed by the customer organization (that participates in the VO itself), and a construction office.

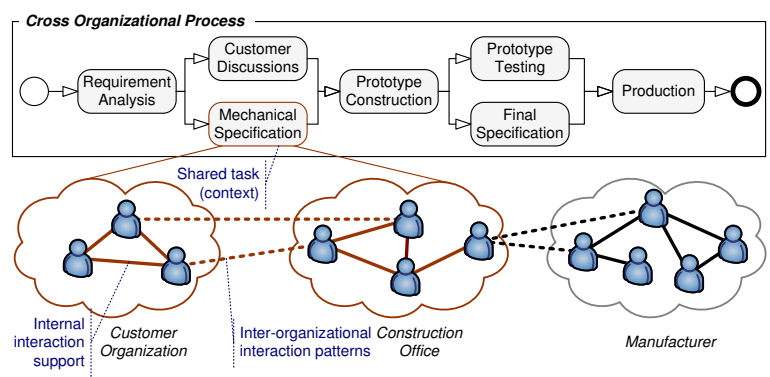

Figure 1. Human interactions in processes spanning organizations.

Consider the following scenario in Figure 1: The construction office creates the mechanical specification for a part required by a customer. However, there are various open issues regarding the efficient production later on. Therefore, an immediate discussion between the customer who has certain requirements, the construction office that designs the prototype, and the manufacturer who is able to optimize the manufacturing process, is needed. Fundamental problems in this scenario include: Who are the persons with the required knowledge in the respective organizations? How can they be contacted, quickly informed about the problem, and involved in discussions? Who are distinguished third party experts that could assist to come to an agreement? What persons can be trusted to make the right decisions as they may have dealt with similar situations before? 
This paper comprises the following contributions. We highlight our novel context-aware human interaction support in process-oriented enterprise collaborations. Therefore, we introduce the $\mathrm{COIN}^{1}$ architecture, as well as the fundamental human interaction concepts. Furthermore, we deal with an expert ranking approach and demonstrate the integration of our work in a typical production planning environment.

\section{RELATED WORK}

Various EU projects have been devoted to support collaborations among people, teams, or companies, such as inContext ${ }^{2}$, ECOSPACE $^{3}$, and ECOLEAD ${ }^{4}$. Based on the work performed in these projects, the goal of COIN is to use the results and provide one unified supporting platform for enterprise collaboration and integration. In addition to prior work, COIN enables context-aware interactions and flexible involvement of humans in complex and highly dynamic networks of enterprises.

In collaborations, activities are the means to capture the context in which human interactions take place. People interact in the context of activities to successfully accomplish their goals. Studies regarding activities in various work settings are described in [3]. They identify patterns of complex business activities, which are then used to derive relationships and activity patterns; see [4] [5] and [6]. Prior work dealing with the management of cross-organizational processes can be found for instance in [7].

For the last years, context has been at the center of many research efforts. In computer science the definition given by Abowd et al. [8] is amongst the most adopted ones.

Multi-criteria decision making and ranking, as surveyed in [9], is used to identify "best" available collaboration partners (experts) when accounting for several input metrics. In particular, we make use of the Promethee approach [10].

Trust relying besides explicit ratings, on monitoring and analyzing interactions and behavior of actors, can be one criterion to rank collaboration partners in social networks. The application of trust relations in team formations and virtual organizations has been studied in [2] [11] and [12].

\section{FOUNDATIONAL CONCEPTS}

Before we deal with our approach to flexible human involvement in cross-organizational processes, we outline the COIN project that represents the underlying basis for our work.

\section{A. The COIN Enterprise Collaboration Architecture}

The COIN project aims at providing an open, selfadaptive integrative solution for Enterprise Interoperability

\footnotetext{
${ }^{1}$ EU FP7-216256 project "COIN": http://www.coin-ip.eu

${ }^{2}$ http://www.in-context.eu

${ }^{3}$ http://www.ip-ecospace.org

${ }^{4}$ http://ecolead.vtt.fi
}

and Enterprise Collaboration. Service orientation is a wellsuited and widely adopted concept in collaboration scenarios, therefore, COIN utilizes state of the art SOA concepts, including Semantic Web Technologies and Software-as-aService (SaaS) models (see [13] for more details). With respect to Enterprise Collaboration, COIN supports numerous features that focus on product development, production planning and manufacturing, and project management in networks of enterprises. As a fundamental aspect, human interactions exist in all forms and phases of virtual organizations and play a major role in the success of collaborations within enterprise networks. Therefore, understanding human interactions and providing advanced support for efficient and effective interactions, is one of the key objectives in COIN's Enterprise Collaboration research track.

The COIN Framework (see Figure 2) consists of (i) the Collaboration Platform (CP) that provides fundamental features that are required in (nearly) every collaboration scenario, and (ii) the Generic Service Platform (GSP) that allows extensions with services following the SaaS model from third party providers. The $\mathrm{CP}$ is designed for and tightly coupled to a Liferay ${ }^{5}$ community portal that provides an effective way to configure and personalize the $\mathrm{CP}$ for specific end-users by providing customized services and tools. Single sign-on- and security mechanisms span services and tools across layers. The GSP relies on semantic web technologies, implemented by the $\mathrm{WSMX}^{6}$ environment and is utilized to discover, bind, compose, and use third-party services at run time.

Because of its extensibility and configurability, the COIN platform can be applied in a wide variety of different collaboration scenarios, ranging from traditional production planning to social campaigning and interest group formations in professional virtual communities. For enabling contextaware interactions, the following baseline components are of major interest (i) user data, including skills and interest profiles, (ii) context data, such as current ongoing activities and user preferences, (iii) integrated baseline services for communication and coordination (e.g., e-mail notifications, and instant messengers), (iv) the GSP as the platform to host extended human interaction services.

\section{B. Process Models for Virtual Organizations}

COIN collaborative Production Planning Services realize innovative solutions in the field of production planning. The C3P (Collaborative Production Planning Platform) service is a graphic environment focusing on collaborative creation of production processes. Companies can conveniently plug themselves to the system, invite potential partners and contribute to the definition of the entire production plan. Furthermore, they collaboratively solve arising problems

\footnotetext{
${ }^{5}$ Open Source Portal Liferay: http://www.liferay.com

${ }^{6}$ WSMX: Web Services Modelling eXecution: http://www.wsmx.org
} 


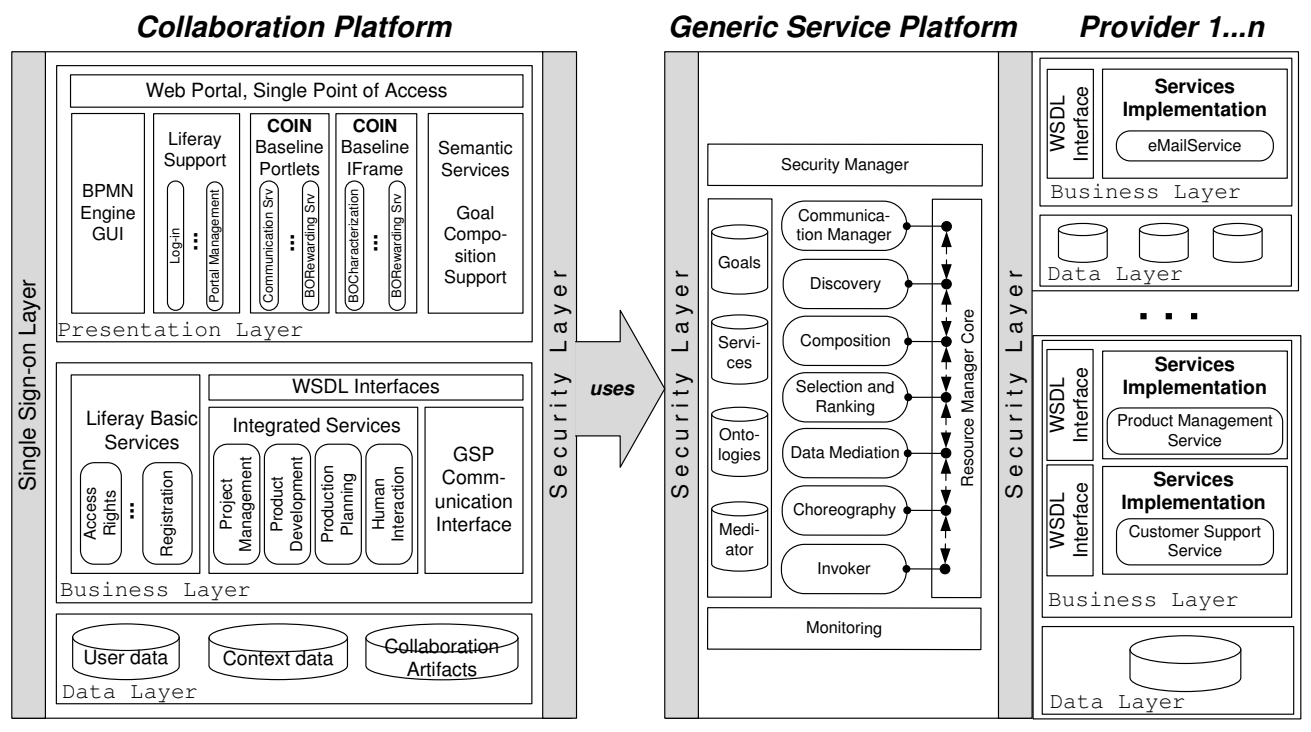

Figure 2. The COIN Framework enabling cross-organizational collaborations.

during the execution using human interaction services. The flow of steps to manufacture a product is defined on two different workflow levels:

Collaborative Public Processes are defined as $\mathrm{XPDL}^{7}$ workflows; whereas each step has at least one responsible partner assigned. The process steps are defined collaboratively by partners, and represent interfaces that hide company-internal (sub-)processes. The Collaborative Public Process Management Service allows multiple users to modify the same process at the same time.

Private Processes define workflows on company-level. Each company describes and imports its own private (sub-)processes involving company resources, such as personnel, material, and machines. Starting from a step of the public process this module allows a particular company to connect its private processes to address the goal of the related public process. Because of privacy issues and protecting know-how, private processes are available to persons of the owning company only.

The meeting points among different partners participating in the collaborative public process are virtual rooms that are linked to the arrows of the workflow model (see Figure 3(a) and 3(b)). Actors can collaboratively define interfaces between process steps, e.g., regarding the shipment of goods. Furthermore, they solve arising problems to find a final agreement of the production plan. Figure 3(a) depicts a public process. Following the BPMN ${ }^{8}$ standard the horizontal white areas represent the customers, the OEM (first tier of the supply chain) and the different suppliers (second supply chain tier). The yellow boxes reflect the public view on the steps of the chain to complete the final item manufactur-

\footnotetext{
${ }^{7}$ XPDL: XML Process Definition Language: http://www.wfmc.org/xpdl.html

${ }^{8}$ BPMN: Business Process Modelling Notation: http://www.bpmn.org
}

ing. Temporal dependencies of activities are clearly visible through the arrows linking the public activities that compose the public process. For the sake of simplicity not all activities are represented in this example.

\section{Human-Interaction Support in Virtual Organizations}

Virtual Organizations pose additional challenges to human interaction support. VOs are typically temporary alliances that form and dissolve again. Various actors are involved in such VOs collaborating and working on joint activities. However, finding the right person to work on tasks or to solve emerging problems is challenging due to scale (number of people involved in VOs) and the temporary nature of formations. Furthermore, actor skills and competencies evolve over time requiring dynamic approaches for the management of these actor properties. In this work, we propose contextaware techniques and tools to address fundamental issues in such collaboration environments: how to find the right person and collaborate with that person using the best suitable communication and interaction channel? We propose the following concepts to address the need for context-aware interactions in VOs:

- Mining of interactions to determine patterns, for example delegation patterns, user preferences, and user behavior (described by multiple metrics).

- Managing context information to select suitable interaction and communication channels.

- Trust inference methods based on social relations to influence communication patterns [2].

Furthermore, human interactions need to be supported in service-oriented systems. Using traditional communication tools (e.g., e-mail, instant messaging tools, or Skype) only is not well suited for that purpose, especially when neglecting context. To address human interactions in SOA, 


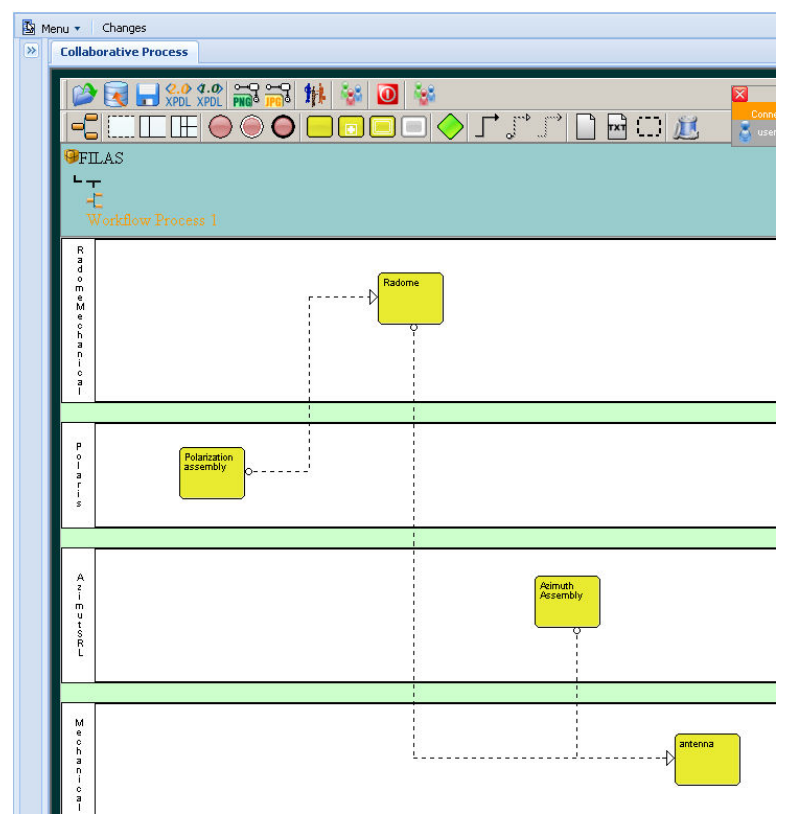

(a) Collaborative Process Design: yellow boxes represent public views on activities of the companies, including items manufacturing or testing; in some cases goods shipping are included in the item manufacturing operation.

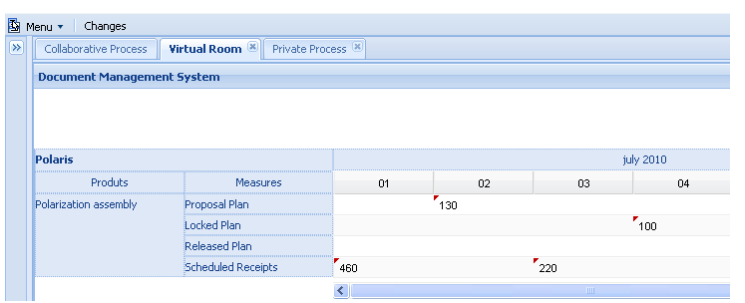

(b) Process Execution Monitoring: visualize planned and actual task execution progress.

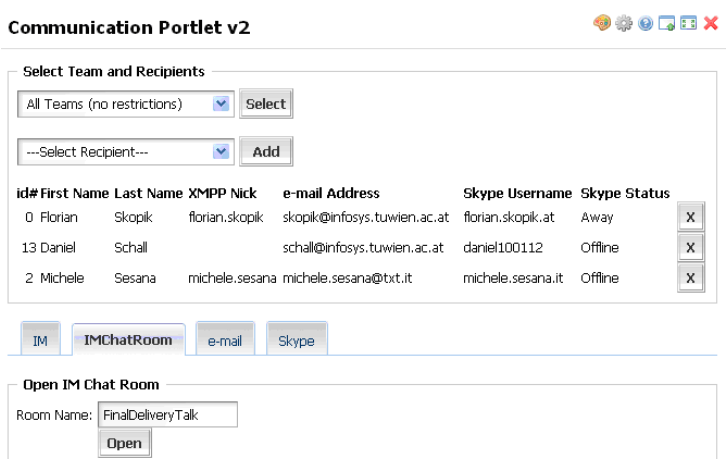

(c) Context-aware Expert Involvement: contact the best experts in their fields considering contextual constraints including communication channels and online presence.

Figure 3. Flexible expert involvement in running processes.

we propose Human-Provided Services [14] that can be utilized for providing "trusted online help and support" enabling experts to define services based on their skills. This approach makes the flexible involvement in workflows possible without designing such interactions a-priori. A set of tools and services support human interactions including: (i) Communication services: chat, e-mail, IM, Skype, and various notification services (ii) Activity management service managing flexible collaboration structures and artifacts used in collaborations (documents, resources, etc.); (iii) Profile management service for storing user skills and capabilities

Specifically, context is used in various ways to support adaptive communication and collaboration: (i) Communication channels can be pre-selected based on user presence (online status), privacy rules, and urgency. (ii) Users are assigned to activities based on their skills but also social preferences of other users working on joint activities. (iii) Expert finding based on reputation in a certain field, for example, with respect to activities that need to be performed.

\section{Context-aware Human Interaction Support}

We highlight our expert query and ranking approach and demonstrate its application in the process-oriented crossorganizational collaboration environments.

\section{A. Context Model}

Observing and mining interactions enables the calculation of metrics that describe the collaboration behavior of network members, including availability, responsiveness, and experience regarding certain activities. Furthermore, mining social network data determines reputation of actors and their trust relations (such as in friend networks). Figure 4 shows the context model, centering all data about actors, relations, resources, etc., around activities. This model is the basis for ranking and selecting experts.

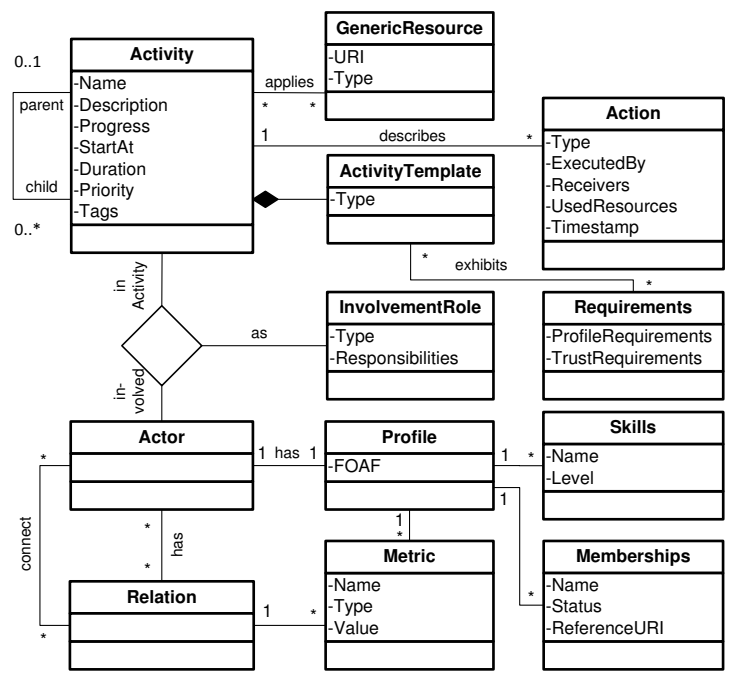

Figure 4. Simplified collaboration context model.

\section{B. Expert Ranking and Query Mechanisms}

We rank members and determine experts with the Promethee approach [10] based on multiple criteria, obtained 


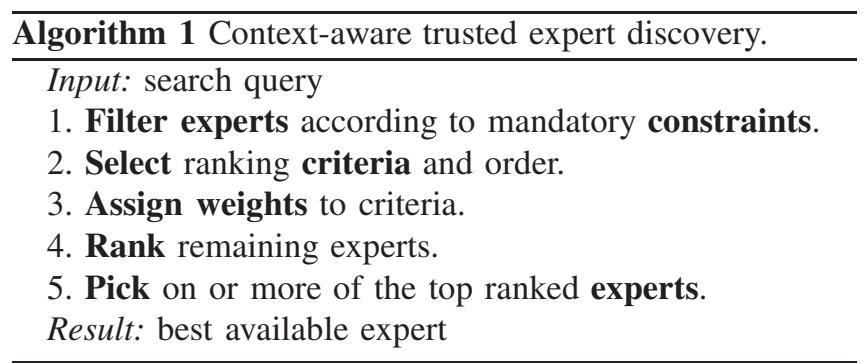

from mining interactions as mentioned above. Our overall approach to determine the best available expert(s) on request is depicted in Algorithm 1.

First (1), all experts that do not fulfill certain constraints, mandatory to support a given activity, e.g., online state, company membership, are sorted out. Afterwards (2), the activity leader sets ranking criteria and their order, for instance experience $\succ$ reputation $\succ$ responsiveness. The order influences the importance (weights) of criteria (3). Then the actual ranking is performed (4), and from the resulting list experts are manually picked (5).

We denote $P_{j}\left(e_{1}, e_{2}\right) \in[0,1]$ as the priority of the choice $e_{1}$ over $e_{2}$ with respect to criteria $j$. For instance, expert $e_{1}$ is preferred over $e_{2}$ regarding metric experience. Since we rank experts with respect to multiple criteria, i.e., values of $k$ metrics, we aggregate priorities as shown in Eq. (1). The weight $w_{j}$ of each criterion $j$ is derived from the specified order of important metrics in the search query.

$$
\pi\left(e_{1}, e_{2}\right)=\sum_{j=1}^{k} P_{j}\left(e_{1}, e_{2}\right) w_{j}
$$

Outrankings (Eq. (2), (3)) compare a choice of an expert $e_{1}$ with the $n-1$ other choices in the set of all available experts $E$. The positive outrank $\Phi^{+}$determines how $e_{1}$ is outranking all other experts, and $\Phi^{-}$determines how all other experts are outranking $e_{1}$. The higher the value of $\Phi^{+}$, and the lower the value of $\Phi^{-}$, the better is the choice $e_{1}$.

$$
\begin{aligned}
\Phi^{+}\left(e_{1}\right) & =\frac{1}{n-1} \sum_{e_{x} \in E} \pi\left(e_{1}, e_{x}\right) \\
\Phi^{-}\left(e_{1}\right) & =\frac{1}{n-1} \sum_{e_{x} \in E} \pi\left(e_{x}, e_{1}\right)
\end{aligned}
$$

Finally, the score of an expert is calculated by Eq. (4).

$$
\Phi\left(e_{1}\right)=\Phi^{+}\left(e_{1}\right)-\Phi^{-}\left(e_{1}\right)
$$

We demonstrate the application of the described Promethee approach [10] with a short example. Assume we rank experts according to different metrics experience, reputation, responsiveness $(\in[0,100])$ with two different queries $Q_{1}=\{\exp \succ$ rep $\succ$ resp $\}$ and $Q_{2}=\{$ resp $\succ$
Table I

EXAMPLE RANKINGS ACCOUNTING FOR EXPERIENCE (exp), REPUTATION ( $r e p$ ), AND RESPONSIVENESS ( $r e s p$ ).

\begin{tabular}{|l|l|l|l|l|l|}
\hline expert & exp & rep & resp & $\Phi_{Q_{1}}($ rank $)$ & $\Phi_{Q_{2}}$ (rank) \\
\hline \hline$e_{1}$ & 50 & 50 & 50 & $1.5(\mathrm{r} 3)$ & $3(\mathrm{r} 1)$ \\
$e_{2}$ & 75 & 25 & 25 & $2(\mathrm{r} 1)$ & $0.5(\mathrm{r} 3)$ \\
$e_{3}$ & 100 & 0 & 0 & $1.75(\mathrm{r} 2)$ & $-3.5(\mathrm{r} 5)$ \\
$e_{4}$ & 0 & 100 & 0 & $-1.75(\mathrm{r} 4)$ & $-1.75(\mathrm{r} 4)$ \\
$e_{5}$ & 0 & 0 & 100 & $-3.5(\mathrm{r} 5)$ & $1.75(\mathrm{r} 2)$ \\
\hline
\end{tabular}

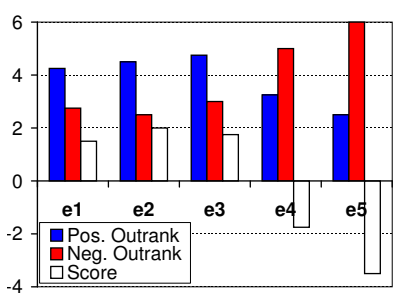

(a) $Q_{1}=\{\exp \succ r e p \succ r e s p\}$.

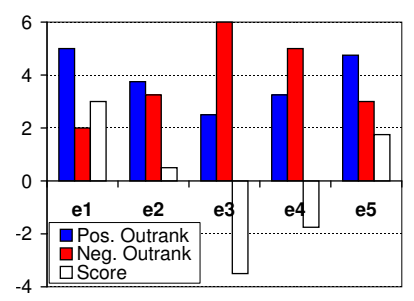

(b) $Q_{2}=\{r e s p \succ r e p \succ e x p\}$.
Figure 5. Ranking results for $Q_{1}$ and $Q_{2}$.

rep $\succ e x p\}$. Table I compares the ranking results, and Figure 5 visualizes expert ranks. Note, the impact of $k$ metrics vary with their position in the queries, and weights are defined as $w_{j}=2^{k-p o s(j)}$.

\section{Applications}

We outline flexible expert involvement and management of communication among two different companies in a shared workflow. The following COIN software modules are used for flexible human interactions in processes:

- COIN baseline including a central database to store and manage profiles of individuals, teams and organizations.

- Activity and task models that are used to infer the context of ongoing work. This information improves the expert search by accounting for experience and expertise.

- C3P production planning software, utilizing concepts of public and private workflows presented before.

- COIN baseline communication services to actually involve experts via e-mail, instant messaging, or Skype.

Figure 3 depicts the single steps of involving experts. In (a), still in the planning phase, partners can be involved to discuss the planned process, while in (b) the actually executed state and emerging problems are discussed. For that purpose, contextual information is derived from a task's execution, including its type, temporal constraints, and the owning company, to discover assistance. This means, the requester for an expert, i.e., the activity owner, can specify an expert search query according to external constraints; for instance, urgent support needs an expert to be currently online and highly responsive; or tasks carrying company sensitive information should not be shared with external people. 
Example Scenario. A manufacturer from China and an assembler from Italy work together on the assembly of a product. The manufacturer in China has to send goods to the company in Italy. Unforeseen problems may happen at China's customs when exporting certain parts. In this case persons from both companies can collaborate in the virtual room (see 3(b)), sharing the current and the adapted production plan, uploading documents from China's custom office, chatting or talking via Skype to find a solution. When the required set of skills, such as far-east custom policies expertise, are not satisfied, third-party experts from outside the currently executed process can be involved through an expert search query. The discussion participants in the virtual room can decide about useful contextual constraints and discover a set of people who match the search query. Finally, the expert requester(s) may pick one or more people to be contacted (visualized in Figure 3(c)).

\section{RESULTS AND FINDINGS}

After extensive discussions with COIN end-user partners, such as Poyry ${ }^{9}$, the system is applied in their business cases. The following results can be mentioned: (i) Enhanced expert discovery mechanisms. By considering not only static competencies, such as official certificates and education, but also dynamically changing experiences, experts can be selected more precisely; especially when accounting for particular contextual constraints, such as online presence for immediate responses or organizational memberships. (ii) Significantly reduced response times. By automatically selecting preferred communication channels, experts can be faster involved in ongoing collaborations. Communication channels are selected based on working time, location of people, and their current activities (all information from the context model). (iii) Harnessing distributed expertise. Involving experts from various physical companies in the same virtual organization massively extends the pool of available skilled persons who can assist in ongoing collaborations.

Besides these positive aspects, we will conduct further research to deal with negative side effects, such as (i) Privacy concerns due to monitoring and mining interactions, (ii) Complex adaptations and extensions of the context model to suitably reflect the real environment.

\section{CONCLUSION AND OUTLOOK}

In this paper we highlighted our novel concepts of context-aware human interactions in cross-organizational processes. We discussed the major building blocks of our solution and demonstrated its application in the COIN project. Discussions with the end-users of COIN software pointed us to their actual daily problems and business challenges. Hence, besides academic concepts addressing context modeling, interaction mining, and expert ranking, we

\footnotetext{
${ }^{9}$ http://www.poyry.com
}

also provide the implementation of our solution addressing real user needs.

\section{ACKNOWLEDGEMENTS}

This work has been partly funded by the European Commission through the project COIN (No. ICT-2008-216256).

\section{REFERENCES}

[1] L. M. Camarinha-Matos and H. Afsarmanesh, "Collaborative networks - value creation in a knowledge society," in Prolamat, 2006.

[2] F. Skopik, D. Schall, and S. Dustdar, "Trusted interaction patterns in large-scale enterprise service networks," in Euromicro PDP, 2010.

[3] B. L. Harrison, A. Cozzi, and T. P. Moran, "Roles and relationships for unified activity management," in GROUP, 2005, pp. 236-245.

[4] T. P. Moran, A. Cozzi, and S. P. Farrell, "Unified activity management: Supporting people in e-business," Communications of the ACM, vol. 48, no. 12, pp. 67-70, 2005.

[5] P. Moody, D. Gruen, M. J. Muller, J. C. Tang, and T. P. Moran, "Business activity patterns: A new model for collaborative business applications," IBM Systems Journal, vol. 45, no. 4, pp. 683-694, 2006.

[6] J. Breslin, A. Passant, and S. Decker, "Social web applications in enterprise," The Social Semantic Web, vol. 48, pp. 251-267, 2009.

[7] Y. Hoffner, H. Ludwig, P. W. P. J. Grefen, and K. Aberer, "Crossflow: integrating workflow management and electronic commerce," SIGecom Exchanges, vol. 2, no. 1, pp. 1-10, 2001.

[8] G. D. Abowd, A. K. Dey, P. J. Brown, N. Davies, M. Smith, and P. Steggles, "Towards a better understanding of context and context-awareness," in International Symposium on Handheld and Ubiquitous Computing, 1999, pp. 304-307.

[9] J. Figueira, S. Greco, and M. Ehrgott, Multiple criteria decision analysis: state of the art surveys. Springer, 2005.

[10] J. Brans and P. Vincke, "A preference ranking organisation method," Management Science, vol. 31, no. 6, pp. 647-656, 1985.

[11] F. Kerschbaum, J. Haller, Y. Karabulut, and P. Robinson, "Pathtrust: A trust-based reputation service for virtual organization formation," in iTrust, 2006, pp. 193-205.

[12] Y. Zuo and B. Panda, "Component based trust management in the context of a virtual organization," in ACM Symposium on Applied Computing, 2005, pp. 1582-1588.

[13] N. Gold, C. Knight, A. Mohan, and M. Munro, "Understanding service-oriented software," IEEE Software, vol. 21, no. 2, pp. 71-77, 2004.

[14] D. Schall, H.-L. Truong, and S. Dustdar, "Unifying human and software services in web-scale collaborations," IEEE Internet Computing, vol. 12, no. 3, pp. 62-68, 2008. 